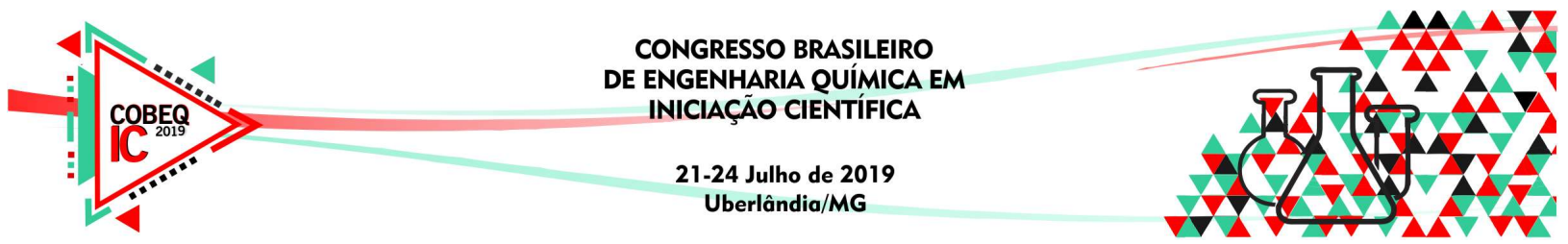

\title{
ESTUDO DA PERFURAÇÃO DE POÇOS DE PETRÓlEO UTILIZANDO A TÉCNICA PRESSURIZED MUD CAP DRILLING: SIMULAÇÃO E EXPERIMENTOS
}

\author{
L. B. ROMUALDO ${ }^{1}$, L. M. ARAUJO ${ }^{1}$, M. A. D. CARVALHO ${ }^{1}$, G. F. M. OLIVEIRA $^{1}$ e M. \\ P. VEGA ${ }^{1}$ \\ ${ }^{1}$ Universidade Federal Rural do Rio de Janeiro, Faculdade de Engenharia Química, \\ Departamento de Engenharia Química \\ E-mail para contato: vega@ufrrj.br
}

\begin{abstract}
RESUMO - A técnica Pressurized Mud Cap Drilling (PMCD) tem sido muito utilizada na perfuração de poços altamente fraturados, visto que esta técnica apresenta um controle preciso frente a extremas perdas de circulação e kicks, em comparação com as técnicas convencionais. O PMCD se dá por meio da injeção de um fluido viscoso na região anular e um fluido de sacrifício na coluna de perfuração. Esta operação não possui retorno para a superfície, o que possibilitou a perfuração de poços antes inviáveis. As principais etapas do $\mathrm{PMCD}$, em um poço que sofreu um kick de gás, consistem, em linhas gerais, na migração de gás com o poço fechado e posterior indução deste fluido invasor para a formação rochosa através da etapa chamada de Bullheading. O Bullheading consiste na injeção de um fluido em contracorrente, para forçar o retorno do gás invasor de volta para o reservatório. O presente trabalho tem como objetivo o estudo da operação de Bullheading durante o uso da técnica PMCD, apresentando resultados de simulação e validação através de experimentos.
\end{abstract}

\section{INTRODUÇÃO}

A fase mais custosa da exploração de petróleo é, geralmente, a perfuração (Vega et al., 2007). Esta etapa necessita de uma operação complexa e robusta para tornar o processo economicamente viável. Para isso, tecnologias precisam ser desenvolvidas constantemente para proporcionar avanços na área, principalmente com a crescente necessidade de se obter petróleo a partir de formações rochosas complexas e desafiadoras (Helgeland, 2014). Os principais avanços nesse quesito foram no conjunto de técnicas MPD (Managed Pressurized Drilling), que procura controlar a pressão anular do poço, baseando-se nos limites de segurança estabelecidos para a operação (Setiawan et al, 2013).

O controle da pressão na região do anular é de extrema importância para o sucesso da perfuração dos poços de petróleo, tendo em vista que o desequilíbrio entre as pressões de fundo do poço, de poros e de fratura do reservatório acarretam em diversos tipos de problemas (Waldmann, 2006). Se a pressão anular de fundo for maior que a pressão de fratura, o fluido terá a tendência de migrar para o reservatório, podendo aumentar as fraturas já existentes. Já o inverso, quando a pressão da formação rochosa é maior que a pressão de fundo, os fluidos nativos do reservatório podem invadir o poço. Esse influxo de fluidos é denominado kick e pode 


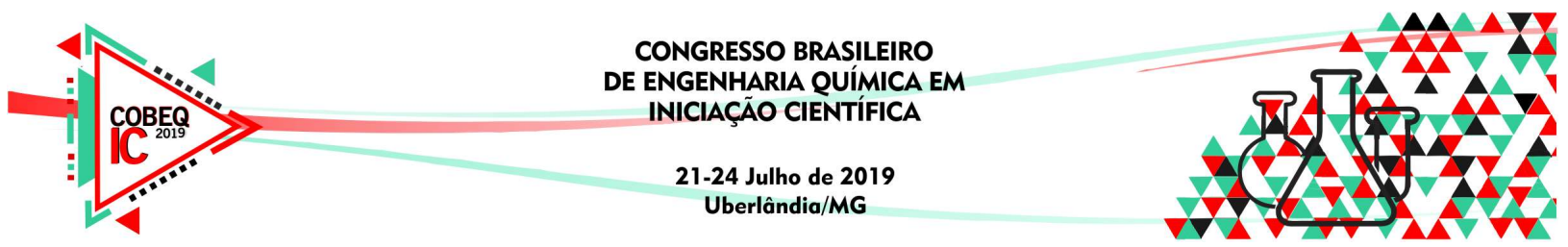

ser controlado ou de dano permanente, podendo levar à desativação do poço ou provocar um blowout (Nygaard, 2006).

A técnica Pressurized Mud Cap Drilling (PMCD) que, como por definição do termo segundo a IADC, é uma variação do Managed Pressurized Drilling (MPD), permite perfurar poços altamente fraturados de forma otimizada, e reduz os riscos ao se trabalhar com os problemas ocasionados por essas fraturas (Setiawan et al, 2013). A operação pode ser definida como um controle da pressão anular de fundo enquanto o poço sofre perdas de circulação e kick, através da injeção de um fluido viscoso na região anular. Este fluido é chamado de lama ou Ligth Annular Mud (LAM), e tem o intuito de impedir a migração do influxo invasor e impulsionar o retorno do mesmo para a formação rochosa. Na coluna de perfuração, bombeiase um fluido de sacrifício (SAC), geralmente água do mar, de forma que este fluido carreie também os cascalhos em direção a formação rochosa (Ghauri, 2014).

O presente trabalho tem como objetivo o estudo sobre a técnica Pressurized Mud Cap Drilling (PMCD) na perfuração de poços de petróleo, apresentando resultados experimentais e simulações.

\section{MATERIAL E MÉTODOS}

A unidade experimental foi construída no Laboratório de Escoamento de Fluidos Giulio Massarani (LEF) do Departamento de Engenharia Química (DEQ), no Instituto de Tecnologia (IT) da Universidade Federal Rural do Rio de Janeiro (UFRRJ), em parceria com a Petrobras.

A unidade experimental dispõe de um tanque com sensor de nível, bomba de deslocamento positivo, tanque de pressão, transdutores de pressão (manométricos e diferenciais), medidor de vazão, válvula de controle choke e outras válvulas manuais e automáticas. Para simular toda a etapa de perfuração utilizou-se somente água como fluido de perfuração e o kick de gás foi simulado utilizando-se ar comprimido. A Figura 1 apresenta o fluxograma da unidade experimental contendo todos estes aparatos citados e a trajetória do fluido no modo convencional MPD (setas azuis), na etapa de bullheading (PMCD) (setas vermelhas) e também a trajetória do gás apresentada pelas setas brancas.

A bomba desloca o fluido do tanque de lama em direção a região anular, quando no meio da trajetória o fluido passa por uma válvula gaveta, que tem como objetivo simular a perda de carga fornecida pela broca numa perfuração real. Assim, o caminho do tanque até a válvula gaveta representa a coluna de perfuração e desta válvula até a válvula choke caracteriza-se a região anular.

O tanque de pressão simula a formação rochosa, e a conexão deste tanque com a região anular se dá pela válvula de permeabilidade, a qual pode-se escolher a abertura de 0 a $100 \%$, possibilitando a simulação de uma formação rochosa com diferentes permeabilidades. A região anular foi construída de forma helicoidal, com angulação diferente de zero para possibilitar a migração de gás, ou seja, evitar que as bolhas ao ascenderem ficassem presas em certas regiões. A unidade experimental representa um poço de 270 metros de profundidade.

A modelagem da operação PMCD foi feita baseada no estudo realizado por Ghauri (2014). O modelo utilizado foi o DFM (Drift flux model), que consiste em um modelo bifásico 

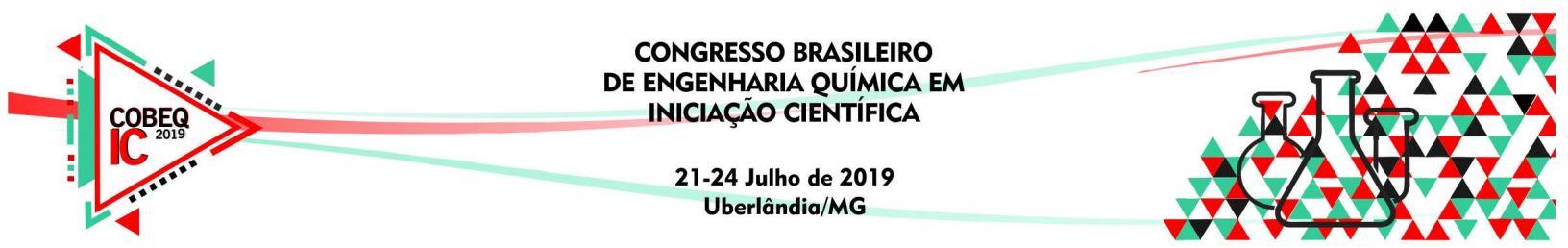

formado pela combinação da equação de conservação de momento da mistura e pelas equações de conservação de massa do gás e do líquido, apresentadas na Equação 1-3, respectivamente (Carvalho, 2018).

Figura 1 - Fluxograma da Unidade Experimental.

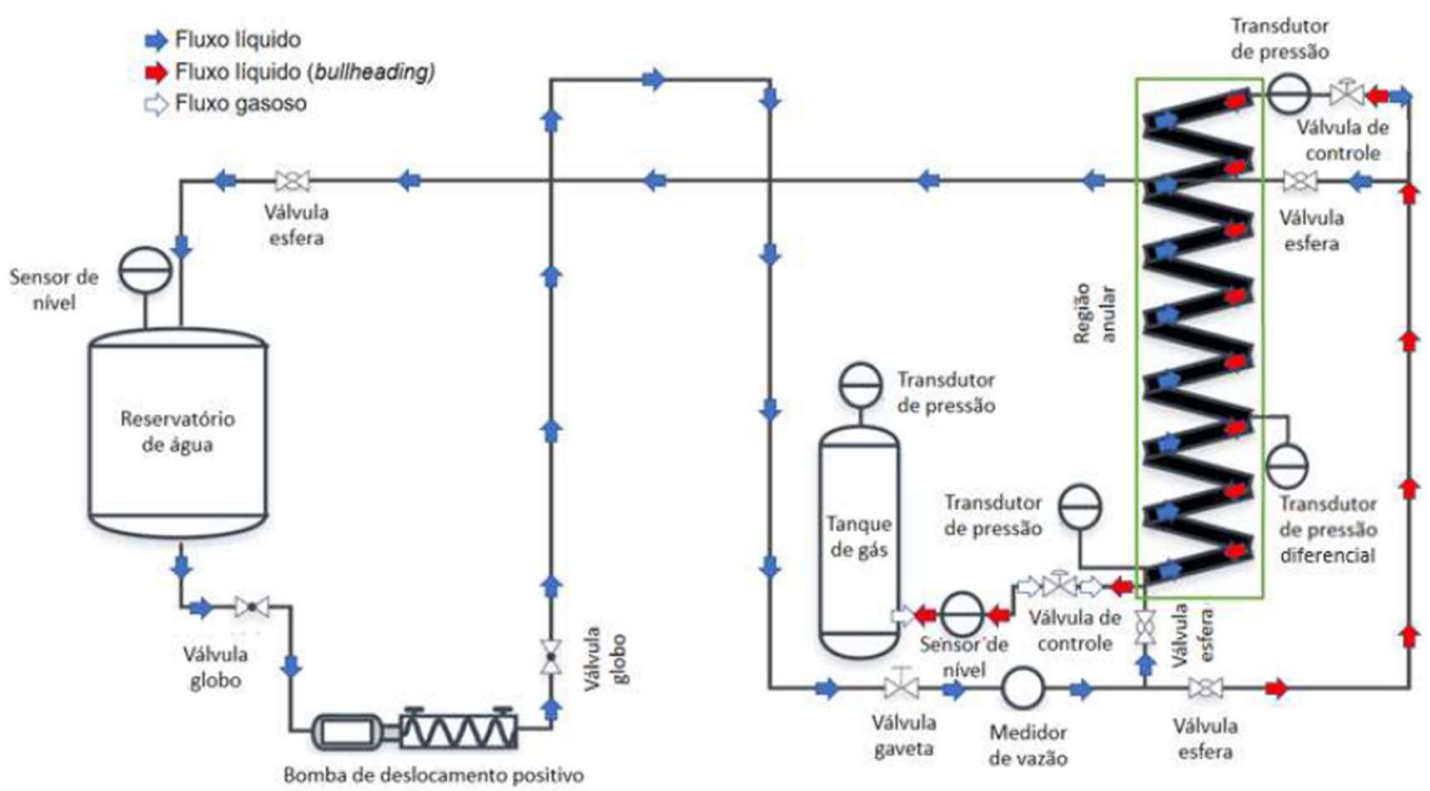

Conservação de momento da mistura:

$\partial_{\mathrm{t}}\left[\mathrm{A}\left(\alpha_{\mathrm{l}} \rho_{\mathrm{l}} v_{\mathrm{l}}+\alpha_{\mathrm{g}} \rho_{\mathrm{g}} v_{\mathrm{g}}\right)\right]+\partial_{\mathrm{x}}\left[\mathrm{A}\left(\alpha_{\mathrm{l}} \rho_{\mathrm{l}} v_{\mathrm{l}}^{2}+\alpha_{\mathrm{g}} \rho_{\mathrm{g}} v_{\mathrm{g}}{ }^{2}\right)\right]+\mathrm{A} \partial_{\mathrm{x}} \mathrm{p}=-\mathrm{A} q$

Conservação de massa da fase gasosa:

$\partial_{\mathrm{t}}\left[\mathrm{A} \alpha_{\mathrm{g}} \rho_{\mathrm{g}}\right]+\partial_{\mathrm{x}}\left[\mathrm{A} \alpha_{\mathrm{g}} \rho_{\mathrm{g}} v_{\mathrm{g}}\right]=\mathrm{A} \Gamma_{\mathrm{g}}$

Conservação de massa da fase líquida:

$\partial_{\mathrm{t}}\left[\mathrm{A} \alpha_{\mathrm{l}} \rho_{\mathrm{l}}\right]+\partial_{\mathrm{x}}\left[\mathrm{A} \alpha_{\mathrm{l}} \rho_{\mathrm{l}} v_{\mathrm{l}}\right]=\mathrm{A} \Gamma_{\mathrm{l}}$

Sendo A a área de escoamento em $m^{2}, \alpha_{l}, \alpha_{g}$ a fração volumétricas de líquido e gás, $\rho_{l}, \rho_{g}$ a densidade do líquido e do gás em $\frac{\mathrm{kg}}{\mathrm{m}^{3}}, v_{l}, v_{g}$ a velocidade do líquido e do gás em $\frac{\mathrm{m}}{\mathrm{s}}, \Gamma_{l}, \Gamma_{g}$ a transferência de massa entre as duas fases em $\frac{\mathrm{kg}}{\mathrm{m}^{3} \mathrm{~s}}, p$ a Pressão em $\mathrm{Pa}$ e $q$ representa as forças de atrito e gravitacional em $\frac{\mathrm{kg}}{\mathrm{m}^{2} \mathrm{~s}^{2}}$.

O modelo é resolvido pelo método numérico AUSMV (Advection Upstream Splitting Method), que consiste em dividir o poço em um conjunto de células, de forma que cada segmento tenha influência nos demais adjacentes. Admite-se então que a célula número 1 representa o fundo do poço e a célula $\mathrm{N}$ o topo do poço, deste modo todas as entradas e saídas se dão nestas células, respectivamente (Carvalho, 2018). 


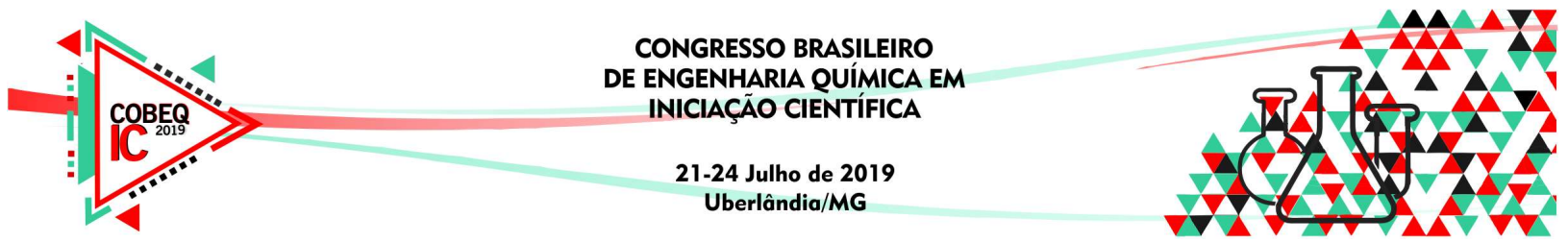

O modelo DFM resulta em equações diferenciais parciais (EDPs) e para a sua resolução o método AUSMV define que os fluxos entre as células dependem do tempo. Segundo Courant, Friedrichs e Lewy para garantir a estabilidade de um método numérico, este deve obedecer a uma condição, que ficou conhecida como condição CFL e está apresentada na Equação 4, e esta deve ser sempre menor que 1 (Carvalho, 2018).

$$
\Delta t \leq \frac{\Delta x}{\operatorname{má} x\left(\left|\lambda_{1}\right|\left|\lambda_{2}\right|\left|\lambda_{3}\right|\right)}
$$

\section{RESULTADOS}

Ghauri (2014) apresenta em seu trabalho estudos da discretização de um poço de 2000 metros de profundidade, mantendo-se satisfeita a condição CFL. Portanto, baseado em seus estudos, nas simulações deste trabalho, o poço foi discretizado em 50 células e fixou-se o intervalo de tempo $\Delta t=0,0005$ segundos.

Primeiro, realizou-se simulações de injeção de gás para análise do comportamento da pressão anular de fundo e da pressão na choke, frente a migração do gás, e validou-se tais simulações a partir de testes experimentais. Os experimentos foram realizados com o índice de abertura da válvula de permeabilidade em $23 \%$ e a pressão do tanque do reservatório controlada em 20 psi, 30 psi, 40 psi, 50 psi, 60 psi, 70 psi, 80 psi e 90 psi, por meio de um controlador feedback PI. Na Figura 2 apresenta-se um resultado típico desta etapa de migração.

Figura 2 - Teste típico para a simulação da etapa de migração.

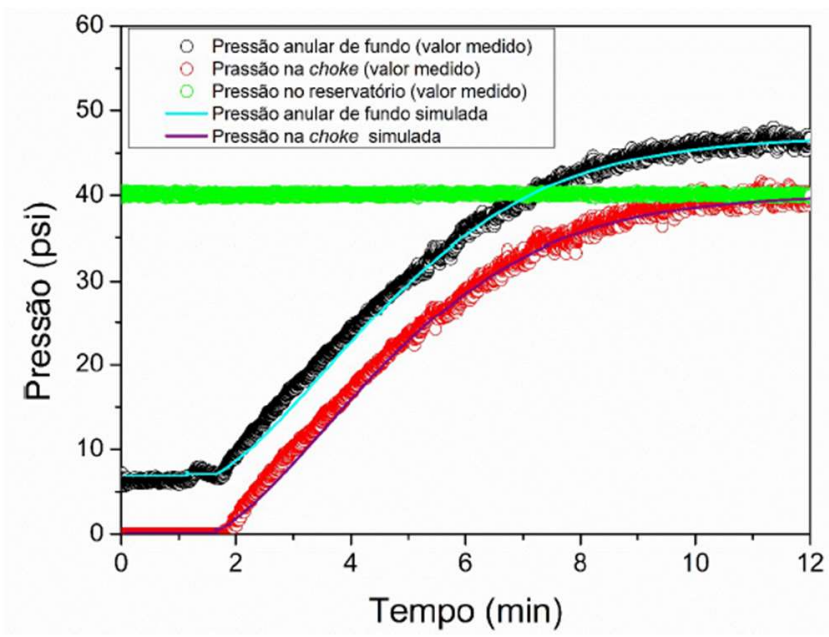

No experimento apresentado na Figura 2, o poço encontra-se aberto e preenchido por água e em 1,15 minuto sofre uma perturbação, um kick de gás, e em 1,65 minuto o poço é fechado. Verifica-se então que a migração de gás após o poço fechado provoca um aumento significativo tanto na pressão na choke quanto na pressão anular de fundo. Além disso, observase que as pressões chegam em um estado estacionário quando a pressão anular se iguala a pressão do reservatório.

Com o intuito de entender melhor o comportamento da pressão na choke durante toda a operação PMCD, realizou-se simulações com injeção e migração de gás e posterior controle 

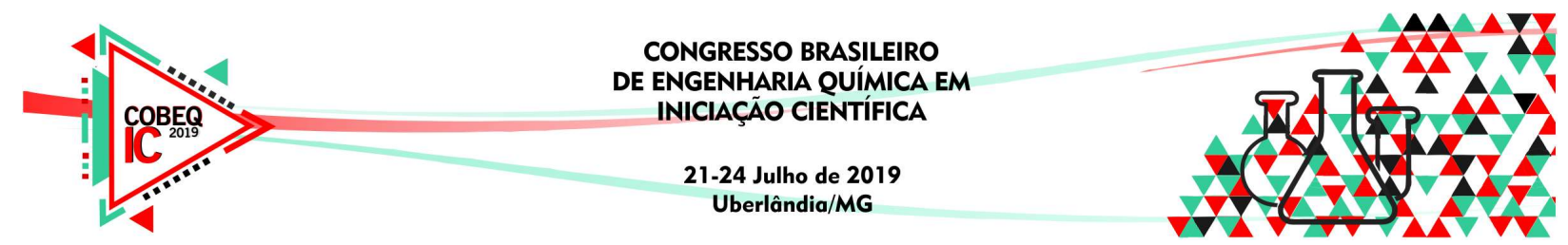

de kick através da operação de Bullheading. Um resultado típico pode ser observado na Figura 3, na qual o distúrbio kick de gás foi implementado em 1,15 min, seguido do fechamento do poço em 2,5 min. O Bullheading se iniciou no minuto 6,6, e terminando no minuto 10 , com frequência da bomba mantida em $13 \mathrm{~Hz}$.

Figura 3 - Validação da simulação da pressão na superfície durante a técnica PMCD.

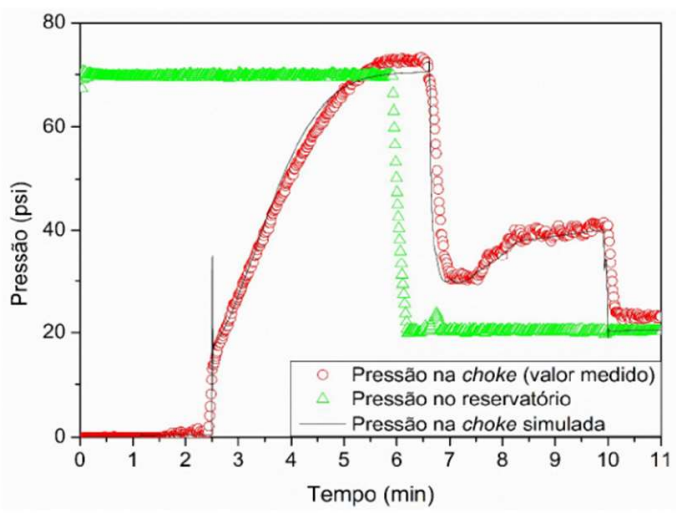

Em seguida, realizou-se testes simulando toda a operação de PMCD. Os experimentos da operação Bullheading foram realizados com injeção de gás, por meio da válvula de permeabilidade, com índice de abertura em $27 \%$ e o tanque reservatório pressurizado em 60 psi, 70 psi ou 80 psi. A implementação do Bullheading, utilizou a frequência da bomba em 13 $\mathrm{Hz}, 15 \mathrm{~Hz}, 17 \mathrm{~Hz}$ ou $20 \mathrm{~Hz}$. A Figura 4 apresenta um típico resultado para um teste experimental da técnica PMCD empregando o tanque reservatório pressurizado em 70 psi.

Figura 4 - Simulação experimental do PMCD.

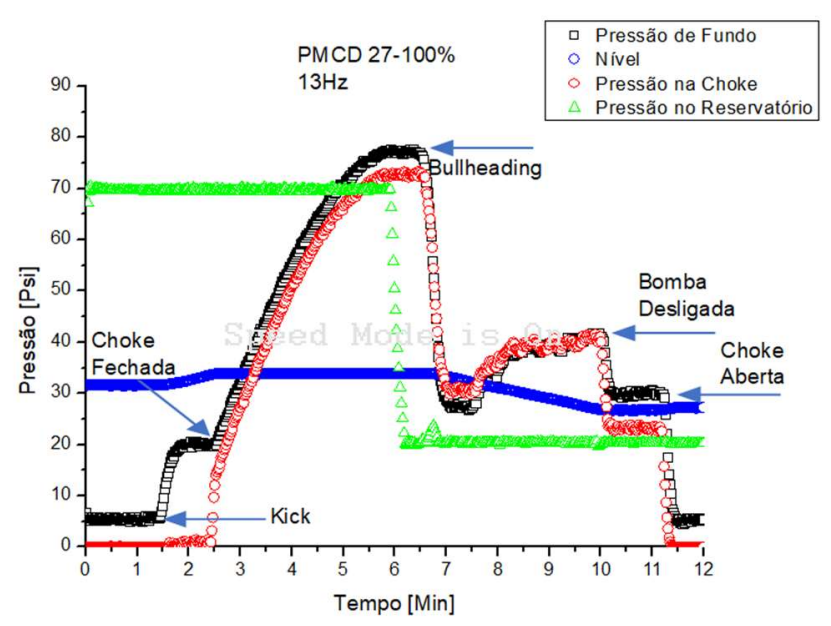

Observa-se na Figura 4 que no instante 1,5 min ocorre o kick de gás, em seguida a migração deste ao longo do anular. Quando atinge certa estabilidade, cerca de 7 minutos, iniciase a etapa de Bullheading com frequência da bomba mantida em $13 \mathrm{~Hz}$, evidenciando uma queda brusca na pressão anular gerada pela expulsão do gás. Entretanto, conforme o anular começa a ser preenchido por líquido novamente, as perdas por atrito aumentam, logo a pressão de fundo sofre um pequeno aumento. Em 10 minutos termina-se o Bullheading, desligando-se 


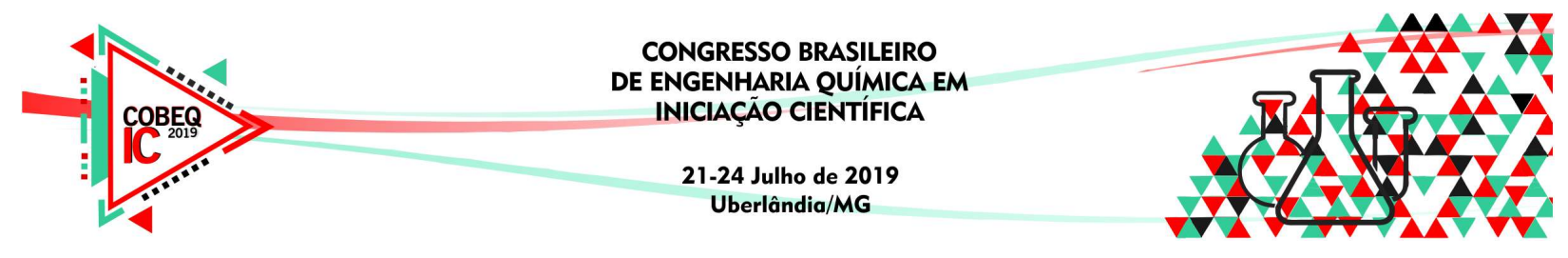

a bomba e em 11 minutos abre-se a choke, ou seja, o poço é aberto e verifica-se que não há mais gás na unidade devido à queda da pressão após a abertura. Se houvesse a presença de gás na unidade, a pressão aumentaria após a abertura da choke.

\section{CONCLUSÃO}

O modelo matemático bifásico desenvolvido conseguiu representar satisfatoriamente toda a operação de Bullheading, apresentando um erro dentro da faixa de $\pm 10 \%$ para a maioria dos valores simulados. As simulações e experimentos conseguiram representar desde o fechamento do poço, após a identificação do kick de gás, a sua posterior migração e seu controle via injeção de fluido em contra fluxo. Através dos dados gerados, foi possível validar o comportamento da pressão de fundo e na choke.

\section{REFERÊNCIAS}

CARVALHO, M. A. D., Estudos Experimentais, de Simulação e de Controle na Perfuração de poços de petróleo utilizando a técnica Pressurized Mud Cap Drilling. Dissertação de Mestrado Programa de Pós-Graduação em Engenharia Química/ UFRRJ. Seropédica, 2018.

COHEN, G.H.; COON, G.A. (1953). Theoretical considerations of retarded control. A.S.M.E., p. $827-834$.

GHAURI, A. A. (2014). Use of the AUSMV scheme for simulation of gas migration, bullheading and Pressurized Mud Cap Drilling, Master's dissertation.

HELGELAND, L.R. (2014). Drilling of Deep-set Carbonates Using Pressurized Mud Cap Drilling. Master's dissertation.

NYGAARD, G., NAEVDAL, G., Nonlinear model predictive control scheme for stabilizing annulus pressure during oil well drilling. Journal of Process Control, 16, 719-732, 2006.

SETIAWAN, B. O., BIN S., BIN, M., FADZLI, N., WUEST, T., (2013). Managed Pressure Drilling with Solids-Free Drilling Fluid Provides Cost-Effective Drilling Solution for Subsea Carbonate Gas Development Wells. IADC/SPE Managed Pressure Drilling and Underbalanced Operations Conference and Exhibition. Society of Petroleum Engineers, IADC/SPE 164573.

VEGA, M.P., MANCINI, M.C., SCHEID, C.M., CALÇADA, L.A., MARTINS, A.L. "Fundamentos de Controle Aplicados à Hidráulica de Perfuração" - ENAHPE, 2007.

WALDMANN, A.; ANDRADE, A.R.; MARTINS A.L.; LOMBA, R.F.T.; ARAGÃO, A.F.L, Sobre os mecanismos responsáveis por minimizar a invasão do fluido de perfuração na rocha reservatório, ENAHPE, Pedra Azul, 2006.

ZIEGLER, J. G. NICHOLS, N. B. Optimum Settings for Automatic Controllers. A.S.M.E. V. 64, p. 759-768, 1942. 